\title{
Detailed Mode of Action of Arabinan-Debranching $\alpha$-L-Arabinofuranosidase GH51 from Bacillus velezensis
}

\author{
Gyo Won $\mathrm{Oh}^{\dagger}$, Yewon Kang ${ }^{\dagger}$, Chang-Yun Choi, So-Yeong Kang, Jung-Hyun Kang, Min-Jae Lee, Nam Soo Han, \\ and Tae-Jip Kim*
}

Division of Animal, Horticultural and Food Sciences, Graduate School of Chungbuk National University, Cheongju 28644, Republic of Korea

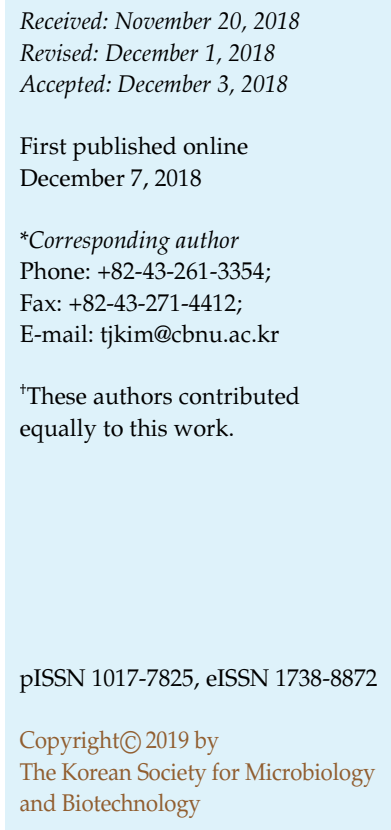

The gene encoding an $\alpha$-L-arabinofuranosidase (BvAF) GH51 from Bacillus velezensis FZB42 was cloned and expressed in Escherichia coli. The corresponding open reading frame consists of 1,491 nucleotides which encode 496 amino acids with the molecular mass of $56.9 \mathrm{kDa}$. BvAF showed the highest activity against sugar beet (branched) arabinan in $50 \mathrm{mM}$ sodium acetate buffer ( $\mathrm{pH}$ 6.0) at $45^{\circ} \mathrm{C}$. However, it could hardly hydrolyze debranched arabinan and arabinoxylans. The time-course hydrolyses of branched arabinan and arabinooligosaccharides (AOS) revealed that BvAF is a unique exo-hydrolase producing exclusively L-arabinose. BvAF could cleave $\alpha$-(1,2)- and/or $\alpha-(1,3)$-L-arabinofuranosidic linkages of the branched substrates to produce the debranched forms of arabinan and AOS. Although the excessive amount of BvAF could liberate L-arabinose from linear AOS, it was extremely lower than that on branched AOS. In conclusion, BvAF is the arabinan-specific exo-acting $\alpha$-L-arabinofuranosidase possessing high debranching activity towards $\alpha$-(1,2)- and/or $\alpha-(1,3)$-linked branches of arabinan, which can facilitate the successive degradation of arabinan by endo- $\alpha-(1,5)-\mathrm{L}-$ arabinanase.

Keywords: Bacillus velezensis, $\alpha$-L-arabinofuranosidase, arabinan-debranching activity, mode of action

\section{Introduction}

As one of the most commonly found pentose sugars in nature, L-Arabinose is abundant as a component of hemicellulosic biomass in plant cell walls. Food scientists in particular are taking an increasing interest in the healthbenefiting functionality of L-arabinose as a low-calorie alternative sweetener [1]. The inhibitory action of L-arabinose against intestinal sucrase is known to reduce the digestion and absorption of sucrose [2]. Recently, the prebiotic effects of arabinoxylan oligosaccharides, linear arabinan, and arabinooligosaccharides (AOS) were also reported on in connection with the growth-promotion of probitic microorganisms such as Bifidobacterium and Lactobacillus species [3-5]. Sugar beet (branched) arabinan is an $\alpha-(1,5)-$ linked L-arabinofuranosyl polymer with $\alpha-(1,2)$ - and/or $\alpha$-(1,3)-linked arabinofuranosyl branches. The cost-effective production of L-arabinose has been accomplished via the enzymatic hydrolysis of sugar beet arabinan by the concerted actions of endo- $\alpha-(1,5)$-L-arabinanase (ABNase; E.C. 3.2.1.99) and exo-acting $\alpha$-L-arabinofuranosidase (AFase; E.C. 3.2.1.55) [6, 7].

The AFase can specifically cleave the terminal nonreducing L-arabinofuranosidic linkages in various arabinosecontaining polymers such as arabinans and arabinoxylans $[8,9]$. For the efficient degradation of heteropolysaccharides such arabinoxylans, AFase should be the essential accessory enzyme which works in concert with other main hemicellulases including $\beta$-xylanase and $\beta$-xylosidase. A variety of bacteria, fungi, and plants have been known as main producers of AFase, which mainly belongs to glycoside hydrolase (GH) families 2, 3, 43, 51, 54, and 62 [10]. Among these six GH families, the AFases GH43 and GH51 include the majority of hydrolases being specific for the degradation of arabinans. The three-dimensional structure of AFase GH51 from Geobacillus stearothermophilus 
revealed that it possesses the catalytic $(\beta / \alpha)_{8}$-barrel domain and the C-terminal jelly-roll architecture [11]. Meanwhile, Streptomyces avermitilis sp. was reported to produce the novel exo-(1,5)- $\alpha$-L-arabinofuranosidase GH43 consisting of the catalytic five-bladed $\beta$-propeller fold with a C-terminal carbohydrate-binding module [12].

The gene clusters involved in the enzymatic degradation and utilization of arabinans and L-arabinose were found from Bacillus subtilis [13, 14], Geobacillus stearothermophilus [15], and Corynebacterium glutamicum [16]. From the genome of B. subtilis $168 \mathrm{~T}^{+}$, two exo-AFases GH51 (AbfA and Abf2) [14] and two endo-ABNases GH43 (AbnA and Abn2) $[17,18]$ were comparatively studied. An intracellular AFase of AbfA is active towards both sugar beet arabinan and debranched arabinan, while the other intracellular enzyme of Abf 2 can hydrolyze only sugar beet arabinan. However, the detailed mode of action of Abf2 has not been reported yet.

In the present study, the putative gene encoding an AFase (hereafter abbreviated as BvAF) was found and cloned from the genome of Bacillus velezensis FZB42. The enzymatic properties and detailed mode of actions towards sugar beet arabinan and branched AOS were investigated by using BvAF as a model enzyme to understand the debranching AFases.

\section{Materials and Methods}

\section{Enzymes and Reagents}

Restriction endonucleases and Pyrobest DNA Polymerase were purchased from Takara Bio (Japan). AccuPrep Plasmid Extraction Kit, PCR purification kit, and oligonucleotide primers were provided by Bioneer (Korea). A DNeasy Blood \& Tissue Kit (Qiagen, Germany) was used for the genomic DNA preparation. Sugar beet arabinan, debranched arabinan, arabinoxylans, and AOS were procured from Megazyme (Ireland).

\section{Gene Amplification and Cloning}

Genomic DNA was prepared from B. velezensis FZB42 (DSM 23117) grown in nutrient broth $(0.5 \%$ peptone, $0.3 \%$ meat extract, and $\left.1.0 \% \mathrm{MnSO}_{4}\right)$ at $30^{\circ} \mathrm{C}$. A set of PCR primers, BvAF-N (5'TTTTGAGCTCATGTCTCACAATACGGC-3') and BvAF-C (5'TTTTCTCGAGAGATGGATCCGCAGG- ${ }^{\prime}$ ), were designed to amplify the gene encoding BvAF (GenBank ID: WP_012118129.1). PCR was performed using a C-1000 Thermal Cycler (Bio-Rad, UK) as follows: an initial denaturation step at $98^{\circ} \mathrm{C}$ for $30 \mathrm{sec}$, followed by 30 cycles consisting of denaturation at $98^{\circ} \mathrm{C}$ for $10 \mathrm{sec}$, annealing at $54^{\circ} \mathrm{C}$ for $30 \mathrm{sec}$, extension at $72^{\circ} \mathrm{C}$ for $1 \mathrm{~min} 30 \mathrm{sec}$, and a final extension at $72^{\circ} \mathrm{C}$ for $5 \mathrm{~min}$. The PCR fragment was digested with NdeI and XhoI, which was cloned into an expression vector, pHCXHD [19]. The resulting recombinant plasmid was designated as pHCBvAF. The entire nucleotide sequence was verified by SolGent (Korea). Escherichia coli MC1061 was used as a host for the gene manipulation and expression.

\section{Gene Expression and Enzyme Purification}

E. coli harboring pHCBvAF was grown in LBA broth $(0.5 \%$ bacto-tryptone, $1 \%$ yeast extract, $1 \% \mathrm{NaCl}, 100 \mu \mathrm{g} / \mathrm{ml}$ of ampicillin) at $37^{\circ} \mathrm{C}$ for $14 \mathrm{~h}$. E. coli BL21 (DE3) harboring pETBIABN [20] was also cultivated in LBA broth at $37^{\circ} \mathrm{C}$ with $0.1 \mathrm{mM} \mathrm{IPTG}$ induction. The recombinant $E$. coli cells were harvested and disrupted by ultrasonicator VCX750 (Sonics \& Materials, USA). Each enzyme with C-terminal six-histidines was purified using Ni-NTA affinity chromatography (Qiagen, Germany). The purity and the molecular mass of each enzyme were determined using 12\% SDS-PAGE analyses. The protein concentration was measured using the BCA protein assay kit (Pierce Biotechnology, USA) with bovine serum albumin as a standard.

\section{Enzyme Activity Assay}

The 3,5-dinitrosalicylic acid (DNS) reducing sugar method [21] was employed for the determination of the hydrolyzing activity against arabinans or arabinoxylans. L-Arabinose/D-Galactose assay kit (Megazyme, Ireland) was applied for the activity assay towards AOS. The activity towards $p$-nitrophenyl arabinofuranoside ( $p$ NPAf; Sigma-Aldrich, USA) was determined by measuring the amount of $p$-nitrophenol liberated from the reaction. The purified enzyme was reacted with $0.5 \%$ of each substrate (except for $2.5 \%$ of arabinoxylans) under the optimal condition for an appropriate reaction time. One unit of enzyme activity on each substrate was defined as the amount of enzyme producing $1 \mu \mathrm{mol} / \mathrm{min}$ of L-arabinose equivalent.

\section{Time-Course Analysis of Hydrolysis Products}

Thin layer chromatography (TLC) was applied to the analysis of hydrolysis products. An appropriate amount of enzyme was reacted with $0.5 \%$ of each substrate under the optimal condition. The resulting hydrolysis products were separated three times on a $60 \mathrm{~F}_{254}$ silica gel plate (Merck, Germany) using chloroform:acetate:water (6:7:1) as a solvent. The spots were visualized by dipping the plate in a developing solution ( $0.3 \% \mathrm{~N}$-1-naphthyl-ethylenediamine and $5 \% \mathrm{H}_{2} \mathrm{SO}_{4}$ in methanol), and subsequently heating it at $110^{\circ} \mathrm{C}$ for $10 \mathrm{~min}$. In order to determine the synergistic arabinan-degradation and hydrolysis yield, $0.5 \%$ of each substrate was reacted with $0.5 \mathrm{U} / \mathrm{ml}$ of BvAF or BlABN (B. licheniformis endo-arabinanase) [20] in $50 \mathrm{mM}$ sodium acetate buffer $\left(\mathrm{pH} \mathrm{6.0)}\right.$ at $45^{\circ} \mathrm{C}$ for $5 \mathrm{~h}$. For the stepwise enzyme treatments, either of exo-BvAF and endo$\mathrm{BlABN}$ was firstly reacted with a substrate for $150 \mathrm{~min}$. After heat inactivation by boiling for $3 \mathrm{~min}$, the next enzyme was then treated for an additional $150 \mathrm{~min}$ at the second step. For the simultaneous enzyme treatment, each substrate was reacted with $0.25 \mathrm{U} / \mathrm{ml}$ of BvAF and BlABN for $300 \mathrm{~min}$. The liberated reducing sugar or L-arabinose was measured by DNS assay and L-Arabinose/D-Galactose assay, respectively. For the time-course 
hydrolysis of branched AOS, $3^{2}-\alpha$-L-arabinofuranosyl-1,5- $\alpha-\mathrm{L}-$ arabinotriose $\left(\mathrm{AA}^{3} \mathrm{~A}\right)$, or the mixture of $3^{2}$ - $\alpha$-L-arabinofuranosyl1,5- $\alpha$-L-arabinotetraose (AAA ${ }^{3} \mathrm{~A}$ ) and $2^{2}, 3^{2}$-di- $\alpha$-L-arabinofuranosyl$1,5-\alpha$-L-arabinotriose $\left(\mathrm{AA}^{2+3} \mathrm{~A}\right)$ was reacted with $0.05 \mathrm{U} / \mathrm{ml}$ of $\mathrm{BvAF}$, respectively. For the complete hydrolysis of AOS, the excessive amount $(0.5 \mathrm{U} / \mathrm{ml})$ of BvAF was treated with a substrate for $12 \mathrm{~h}$.

\section{Results and Discussion}

\section{Gene Cloning and Expression of BvAF}

The Gram-positive bacterium, Bacillus velezensis FZB42 (a synonym of $B$. amyloliquefaciens subsp. plantarum and B. methylotrophicus), was known as the model strain for the promotion of plant growth and the biocontrol of rhizobacteria [22, 23]. Recent comparative genome analyses among various $B$. velezensis spp. have revealed their potential as degraders of lignocellulosic biomass [24]. An open reading frame encoding a novel AFase (BvAF) was found from the genome of $B$. velezensis FZB42 [25]. Approximately $1.5 \mathrm{~kb}$ of DNA fragment was amplified using a set of PCR primers, BvAF-N and BvAF-C. The resulting PCR-fragment was cloned into a constitutive expression vector of $\mathrm{pHCXHD}$, which was designated as $\mathrm{pHCBvAF}$. The DNA sequencing analysis verified that the corresponding open reading frame consists of 1,491 nucleotides encoding 496 amino acid residues. The gene encoding BvAF with C-terminal six-histidines was constitutively expressed from E. coli harboring $\mathrm{pHCBvAF}$ (Fig. 1). The recombinant BvAF was purified to an apparent

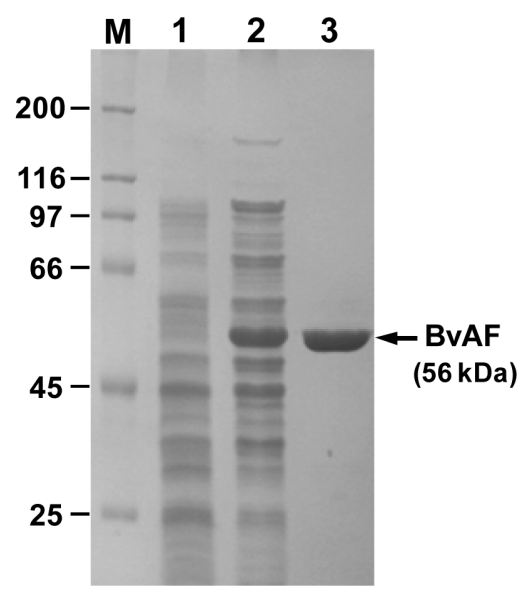

Fig. 1. SDS-PAGE analysis for gene expression and enzyme purification of recombinant BvAF from E. coli.

Lane $\mathrm{M}$, protein size marker; 1, cell extract from E. coli harboring an empty vector (pHCXHD); 2, cell extract from E. coli harboring pHCBvAF; 3, recombinant BvAF purified by Ni-NTA chromatography. homogeneity via Ni-NTA affinity chromatography. The apparent molecular mass of recombinant BvAF including six-histidines is approximately $56 \mathrm{kDa}$, which coincides with its calculated molecular mass of 56,857 Da.

Two genes encoding AFases GH51 with different substrate specificities, AbfA (WP_004398747.1) and Abf2 (WP_004398654.1), were previously found from the genome of $B$. subtilis $168 \mathrm{~T}^{+}$[14]. The deduced amino acid sequence of BvAF shares identities of $23.8 \%$ and $81.3 \%$ with AbfA and Abf2, respectively. The AFases from Geobacillus sp. KCTC3012 (ABM68633.1) [19], Geobacillus stearothermophilus T-6 (AAD45520.2) [11], and Thermotoga maritima MSB8 (NP_228093.1) [26] showed 23.3\%, 23.8\%, and 27.5\% of amino acid sequence identities with BvAF, respectively. The overall structure of common AFases GH51 consists of both catalytic $(\beta / \alpha)_{8}$-barrel domain and C-terminal jellyroll domain [11, 27]. Although the primary structure similarity of BvAF with the other AFases GH51 is less than $30 \%$, the amino acid sequence alignment revealed that BvAF shares two putative catalytic amino acid residues, a general acid/base (Glu172) and a nucleophile (Glu295).

\section{Enzymatic Characterization of BvAF}

To optimize the reaction conditions, the hydrolyzing activity of BvAF on sugar beet arabinan as a substrate was determined by using DNS reducing sugar and L-arabinose assay methods. BvAF exhibited the highest activity $(41.3 \mathrm{U} / \mathrm{mg})$ in $50 \mathrm{mM}$ sodium acetate buffer ( $\mathrm{pH}$ 6.0) at $45^{\circ} \mathrm{C}$ (Fig. 2). It also showed over $77 \%$ of relative activity at $35 \sim 55^{\circ} \mathrm{C}$, but it was rapidly decreased to $16 \%$ at $70^{\circ} \mathrm{C}$. The half-life of BvAF was determined as $15 \mathrm{~h}$ at $45^{\circ} \mathrm{C}$ and $4 \mathrm{~min}$ at $70^{\circ} \mathrm{C}$, respectively. BvAF possesses relatively narrow optimum near $\mathrm{pH} 6.0$, but it showed the relatively high $\mathrm{pH}$ stability at the broad $\mathrm{pH}$ range of 4.0 10.0 (data not shown). Most known microbial AFases have their reaction optima at $\mathrm{pH} 5.0 \sim 8.0$ and $40 \sim 60^{\circ} \mathrm{C}[8,9]$. For example, the AFases (AbfA and Abf2) from B. subtilis showed their highest activity at $50 \sim 60^{\circ} \mathrm{C}$ and $\mathrm{pH} 8.0$ [14], whereas that from Geobacillus sp. (GAF) is also highly active at $60^{\circ} \mathrm{C}$ and $\mathrm{pH} 5.0$ [19]. The highly thermostable AFases were also reported from Thermotoga maritima [26] and Caldicellulosiruptor saccharolyticus [28].

The specific activities of BvAF were investigated on various substrates such as $p$-NPAf, AOS, arabinans, and arabinoxylans (Table 1). BvAF showed the highest activity (207.6 U/mg) on $p$-NPAf, the synthetic substrate for exoAFase activity assay, which implies that BvAF is likely to be a typical exo-acting AFase. This enzyme could preferentially hydrolyze sugar beet arabinan, whereas it showed only 

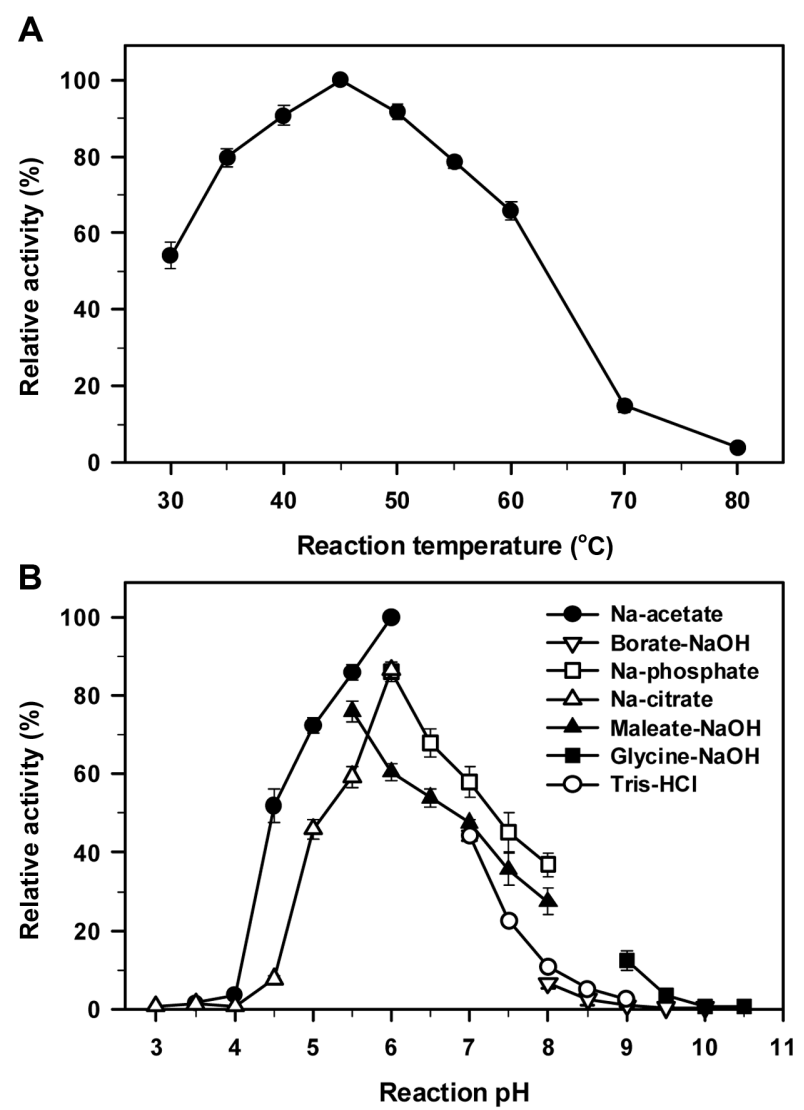

Fig. 2. Effect of (A) temperature and (B) $\mathrm{pH}$ on the arabinanhydrolyzing activity of BvAF.

Relative activities of BvAF on sugar beet arabinan were determined at different temperatures and $\mathrm{pH}$ by using DNS reducing sugar assay.

negligible activities towards various arabinoxylans. Interestingly, BvAF could hardly hydrolyze debranched

Table 1. Specific activities of BvAF on polymeric and oligomeric substrates.

\begin{tabular}{lc}
\hline \multicolumn{1}{c}{ Substrate } & Specific activity $(\mathrm{U} / \mathrm{mg})$ \\
\hline$p$-Nitrophenyl arabinofuranoside & $207.56 \pm 9.70$ \\
\hline Sugar beet arabinan & $41.29 \pm 4.13$ \\
Debranched arabinan & $0.88 \pm 0.14$ \\
Arabinoxylans $^{\mathrm{a}}$ & $\mathrm{ND}^{\mathrm{b}}$ \\
\hline Arabinobiose & $3.10 \pm 0.17$ \\
Arabinotriose & $2.05 \pm 0.22$ \\
Arabinotetraose & $3.09 \pm 0.26$ \\
Arabinopentaose & $3.43 \pm 0.16$ \\
Arabinohexaose & $3.46 \pm 0.21$ \\
\hline
\end{tabular}

${ }^{\mathrm{a} O a t-s p e l t, ~ r y e, ~ a n d ~ w h e a t ~ a r a b i n o x y l a n s ~}$

${ }^{\mathrm{b}}$ Enzymatic activity was not detected.

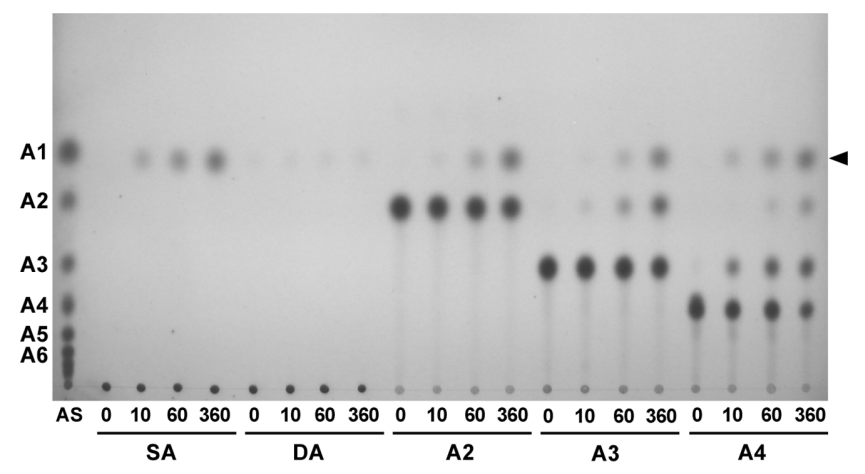

Fig. 3. TLC analysis of the hydrolysates from various substrates reacted with BvAF.

Each substrate $(0.5 \%)$ was reacted with $0.5 \mathrm{U} / \mathrm{ml}$ of BvAF at $\mathrm{pH} 6.0$ and $45^{\circ} \mathrm{C}$ for $0,10,60$, and $360 \mathrm{~min}$. The arrow head indicates L-arabinose as a reaction product. AS, arabinooligosaccharides standard; SA, sugar beet arabinan; DA, debranched arabinan; A2 A4, arabinobiose to arabinotetraose.

arabinan, a common substrate for AFases. Its much lower relative activities (7 9\%) on linear AOS than sugar beet arabinan polymer suggested that BvAF is a novel exohydrolase exclusively cleaving $\alpha-(1,2)-$ and/or $\alpha-(1,3)-$ linked arabinosyl residues at the branches, not $\alpha-(1,5)-$ linkages in the backbone of sugar beet arabinan.

B. subtilis AbfA is the intracellular AFase being active towards both sugar beet arabinan and debranched arabinan, which can be expressed from the gene cluster for arabinandegradation and utilization. On the contrary, the other intracellular Abf2 could hydrolyze only sugar beet arabinan, which is not included within the same gene cluster. As the detailed mode of debranching action of Abf2 has not been reported yet, BvAF was investigated as a model for debranching AFases in the present study.

\section{Detailed Mode of Debranching Action of BvAF}

In order to investigate the hydrolysis patterns of BvAF, $0.5 \mathrm{U} / \mathrm{ml}$ of enzyme was reacted with $0.5 \%$ of each substrate, and the resulting hydrolysates were comparatively identified by TLC analysis (Fig. 3). As the reaction proceeded, BvAF could exclusively release L-arabinose without any AOS intermediates from sugar beet arabinan. The time-course analyses revealed the stepwise degradation of AOS via exotype enzymatic actions of BvAF. For example, BvAF firstly hydrolyzed arabinotetraose to arabinotriose and L-arabinose, and then the resulting arabinotriose was further hydrolyzed into arabinobiose and L-arabinose. However, its much lower activity towards $\alpha$-(1,5)-arabinofuranosyl linkages resulted in the slow and incomplete hydrolysis of linear 
AOS. When the excessive amount $(5.0 \mathrm{U} / \mathrm{ml})$ of BvAF was reacted with the substrates for $12 \mathrm{~h}$, all the AOS substrates were finally hydrolyzed into only L-arabinose (data not shown).

An endo- $\alpha-(1,5)-\mathrm{L}$-arabinanase from $B$. licheniformis DSM 13 (BlABN) was known to have no detectable activity on sugar beet arabinan, due to its extremely low accessibility towards branched structure [20]. Therefore, the synergistic and efficient degradation of sugar beet arabinan was achieved via the simultaneous treatment with an AFase from Geobacillus sp. KCTC3012 (GAF) [7]. As GAF has almost the same activities $(4.52 \mathrm{U} / \mathrm{mg})$ against both sugar beet and debranched arabinans, the simultaneous treatment of both

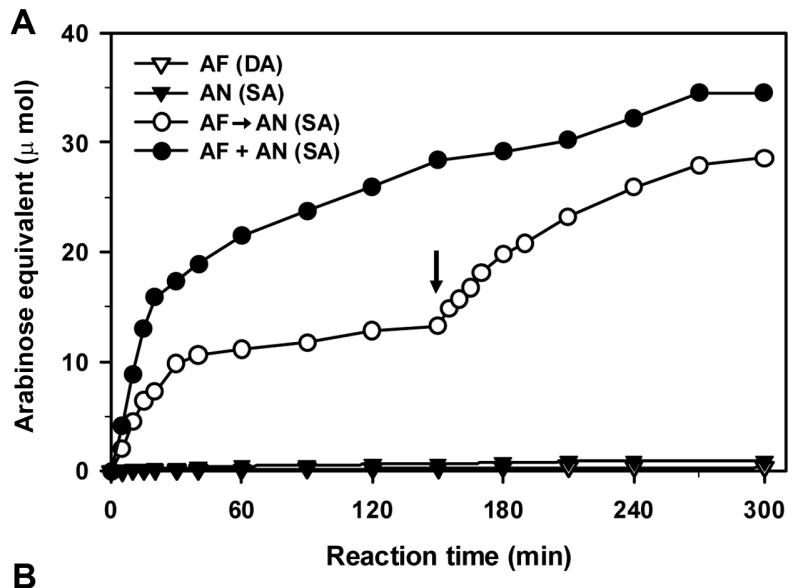

B

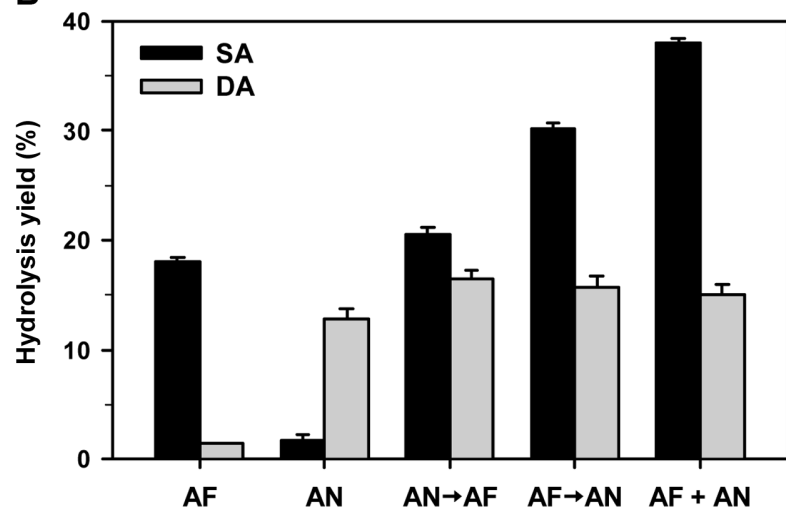

Fig. 4. Time-course analysis of (A) arabinan-degradation and (B) hydrolysis yield via single, stepwise, or simultaneous treatment with BvAF (AF) and BlABN (AN).

$0.5 \mathrm{U} / \mathrm{ml}$ of each enzyme was reacted with $0.5 \%$ of sugar beet arabinan (SA) or debranched arabinan (DA) for $300 \mathrm{~min}$, respectively. For the stepwise treatment, the first enzyme was heat-inactivated after $150 \mathrm{~min}$ (vertical arrow), and the second enzyme was reacted for additional $150 \mathrm{~min}$. For the simultaneous treatment, $0.25 \mathrm{U} / \mathrm{ml}$ of both $\mathrm{AF}$ and $\mathrm{AN}$ were reacted with arabinan for $300 \mathrm{~min}$. The hydrolysis products were measured by DNS reducing sugar assay.
exo-GAF and endo-BlABN on arabinans could maximize their cooperative and complementary actions to shorten the operation time and increase the conversion yield into L-arabinose. The synergistic production of L-arabinose by the thermostable exo- and endo-arabinanases was also reported from Caldicellulosiruptor saccharolyticus [6]. In Fig. 4 , the single treatment of BlABN or BvAF $(0.5 \mathrm{U} / \mathrm{ml})$ showed no detectable activity against sugar beet arabinan or debranched arabinan, respectively. When sugar beet arabinan was pre-treated with BvAF for $150 \mathrm{~min}$, a total of $13.3 \mu \mathrm{mol} \mathrm{L}$-arabinose was released. Interestingly, BlABN could attack the resulting BvAF-treated sugar beet arabinan to produce various AOS up to $28.6 \mu \mathrm{mol}$ of arabinose equivalent. The simultaneous treatment of BvAF and BlABN resulted in more rapid hydrolysis of sugar beet arabinan at the early reaction step than in the single or the stepwise enzyme treatments. The pre-treatment of BvAF
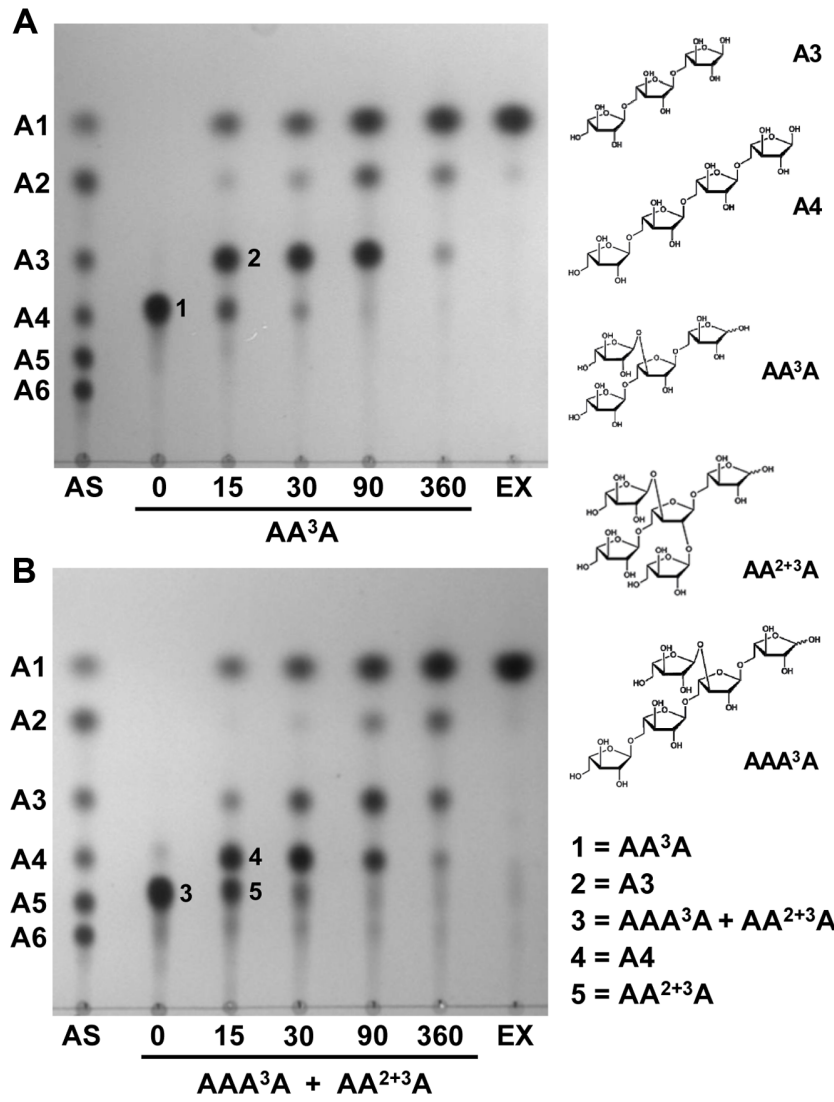

$1=\mathrm{AA}^{3} \mathrm{~A}$

$2=A 3$

$3=A A A^{3} A+A A^{2+3} A$

$4=\mathrm{A4}$

$5=\mathrm{AA}^{2+3} \mathrm{~A}$

Fig. 5. TLC analysis of the hydrolysates from the branched arabinooligosaccharides by BvAF.

(A) $0.5 \%$ of $\mathrm{AA}^{3} \mathrm{~A}$ or (B) the mixture of $\mathrm{AAA}^{3} \mathrm{~A}$ and $\mathrm{AA}^{2+3} \mathrm{~A}$ was reacted with $0.05 \mathrm{U} / \mathrm{ml}$ of $\mathrm{BvAF}$ at $\mathrm{pH} 6.0$ and $45^{\circ} \mathrm{C}$ for $0,15,30,90$, and $360 \mathrm{~min}$, respectively. AS, arabinooligosaccharides standard; EX, the excessive treatment with $0.5 \mathrm{U} / \mathrm{ml}$ of BvAF for $12 \mathrm{~h}$. 
could remove $\alpha$-(1,2)- and/or $\alpha$-(1,3)-linked arabinofuranosyl branches of sugar beet arabinan to generate the debranched form of arabinan, which can be the more accessible substrate for the endo-actions of BlABN. Although the simultaneous treatment of BvAF and BlABN showed the highest hydrolysis yield of $38.1 \%$ from sugar beet arabinan, it is much lower than that with GAF and BlABN (91.5\%) [7]. The incomplete degradation of arabinan into the residual short AOS was caused by the much lower hydrolyzing activity of BvAF against the $\alpha-(1,5)$-linked arabinan backbone.

To understand the detailed mode of debranching actions, three different branched AOS were reacted with $0.05 \mathrm{U} / \mathrm{ml}$ of $\mathrm{BvAF}$, and the resulting hydrolysates were identified by time-course TLC analysis (Fig. 5). In case of $\mathrm{AA}^{3} \mathrm{~A}, \mathrm{BvAF}$ firstly attacked $\alpha$-(1,3)-linked arabinofuranosyl branch to produce arabinotriose. The resulting arabinotriose was very slowly degraded to L-arabinose and arabinobiose. Towards the mixture of $\mathrm{AAA}^{3} \mathrm{~A}$ and $\mathrm{AA}^{2+3} \mathrm{~A}, \alpha-(1,3)$-linked arabinofuranosyl branch of $\mathrm{AAA}^{3} \mathrm{~A}$ was rapidly removed via the debranching activity of BvAF at the early reaction stage. The resulting arabinotetraose was also very slowly hydrolyzed to produce L-arabinose and AOS intermediates. After the preferred hydrolysis of $\mathrm{AAA}^{3} \mathrm{~A}$, the residual $\mathrm{AA}^{2+3} \mathrm{~A}$ was gradually hydrolyzed into arabinotriose at a slow rate. When $0.5 \mathrm{U} / \mathrm{ml}$ of BvAF was reacted for $12 \mathrm{~h}$, all three different branched AOS were completely saccharified into L-arabinose as a sole final product. The detailed mode of action of BvAF on sugar beet arabinan and branched AOS was schematically represented in Fig. 6.

Compared with common AFases including GAF, BvAF is a novel arabinan-specific exo-hydrolase with high
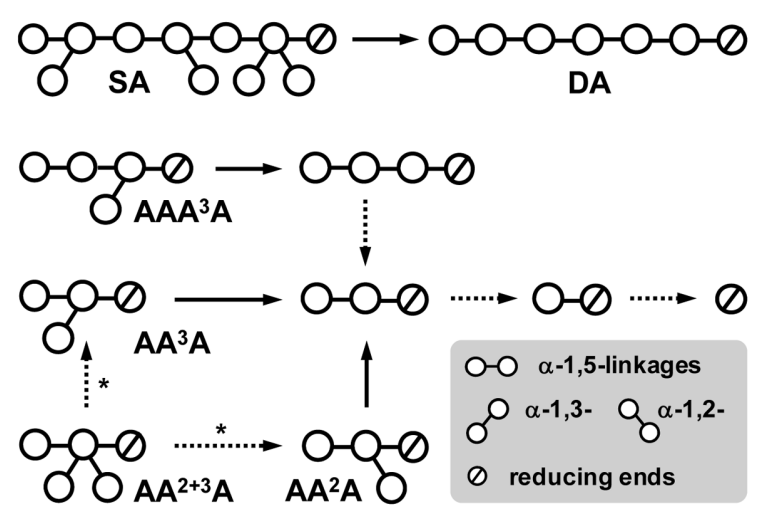

Fig. 6. Proposed mode of debranching actions of BvAF against sugar beet arabinan and branched arabinooligosaccharides. The solid and the dashed arrows indicate the high and the extremely low hydrolyzing activities of BvAF, respectively. The routes marked by asterisks are not clarified. debranching activity towards $\alpha$-(1,2)- and/or $\alpha$-(1,3)-linked branches of sugar beet arabinan to generate the debranched arabinan. The debranching actions of BvAF can promote more rapid and efficient arabinan-degradation by the other exo- and endo-arabinosyl hydrolases from $B$. velezensis. A deeper insight into the AFases with versatile modes of actions will expedite enzyme engineering for the development of designer prebiotics in future.

\section{Acknowledgment}

This work was supported by the National Research Foundation (NRF-2017M3C1B5019292) of the Ministry of Science, ICT and Future Planning, Republic of Korea.

\section{Conflict of Interest}

The authors have no financial conflicts of interest to declare.

\section{References}

1. Hizukuri S. 1999. Nutritional and physiological functions and uses of L-arabinose. J. Appl. Glycosci. 46: 159-165.

2. Seri K, Sanai K, Matsuo N, Kawakubo K, Xue C, Inoue S. 1996. L-Arabinose selectively inhibits intestinal sucrase in an uncompetitive manner and suppresses glycemic response after sucrose ingestion in animals. Metabolism 45: 1368-1374.

3. Grootaert C, Delcour JA, Courtin CM, Broekaert WF, Verstraete W, Van de Wiele T. 2007. Microbial metabolism and prebiotic potency of arabinoxylan oligosaccharides in the human intestine. Trends Food Sci. Technol. 18: 64-71.

4. Vigsnæs LK, Holck J, Meyer AS, Licht TR. 2011. In vitro fermentation of sugar beet arabino-oligosaccharides by fecal microbiota obtained from patients with ulcerative colitis to selectively stimulate the growth of Bifidobacterium spp. and Lactobacillus spp. Appl. Environ. Microbiol. 77: 8336-8344.

5. Moon JS, Shin SY, Choi HS, Joo W, Cho SK, Li L, et al. 2015. In vitro digestion and fermentation properties of linear sugar-beet arabinan and its oligosaccharides. Carbohydr. Polym. 131: 50-56.

6. Lim YR, Yeom SJ, Kim YS, Oh DK. 2011. Synergistic production of L-arabinose from arabinan by the combined use of thermostable endo- and exo-arabinanases from Caldicellulosiruptor saccharolyticus. Bioresour. Technol. 102: 4277-4280.

7. Park JM, Jang MU, Oh GW, Lee EH, Kang JH, Song YB, et al. 2015. Synergistic action modes of arabinan degradation by exo- and endo-arabinosyl hydrolases. J. Microbiol. Biotechnol. 25: 227-233.

8. Saha BC. 2000. $\alpha$-L-Arabinofuranosidases: biochemistry, 
molecular biology and application in biotechnology. Biotechnol. Adv. 18: 403-423.

9. Numan MT, Bhosle NB. 2006. $\alpha$-L-Arabinofuranosidases: the potential applications in biotechnology. J. Ind. Microbiol. Biotechnol. 33: 247-260.

10. Wilkens C, Andersen S, Dumon C, Berrin JG, Svensson B. 2017. GH62 arabinofuranosidases: Structure, function and applications. Biotechnol. Adv. 35: 792-804.

11. Hövel K, Shallom D, Niefind K, Belakhov V, Shoham G, Baasov T, et al. 2003. Crystal structure and snapshots along the reaction pathway of a family $51 \alpha$-L-arabinofuranosidase. EMBO J. 22: 4922-4932.

12. Fujimoto $Z$, Ichinose $H$, Maehara $T$, Honda M, Kitaoka $M$, Kaneko S. 2010. Crystal structure of an exo-1,5- $\alpha$-Larabinofuranosidase from Streptomyces avermitilis provides insights into the mechanism of substrate discrimination between exo- and endo-type enzymes in glycoside hydrolase family 43. J. Biol. Chem. 285: 34134-34143.

13. de Sá-Nogueira I, Nogueira TV, Soares S, de Lencastre H. 1997. The Bacillus subtilis L-arabinose (ara) operon: nucleotide sequence, genetic organization and expression. Microbiology 143: $957-969$.

14. Inácio JM, Correia IL, de Sá-Nogueira I. 2008. Two distinct arabinofuranosidases contribute to arabino-oligosaccharide degradation in Bacillus subtilis. Microbiology 154: 2719-2729.

15. Shulami S, Raz-Pasteur A, Tabachnikov O, Gilead-Gropper S, Shner I, Shoham Y. 2011. The L-arabinan utilization system of Geobacillus stearothermophilus. J. Bacteriol. 193: 2838-2850.

16. Kawaguchi $H$, Sasaki $M$, Vertès AA, Inui $M$, Yukawa $H$. 2009. Identification and functional analysis of the gene cluster for L-arabinose utilization in Corynebacterium glutamicum. Appl. Environ. Microbiol. 75: 3419-3429.

17. Leal TF, de Sá-Nogueira I. 2004. Purification, characterization and functional analysis of an endo-arabinanase (AbnA) from Bacillus subtilis. FEMS Microbiol. Lett. 241: 41-48.

18. Inácio JM, de Sá-Nogueira I. 2008. Characterization of $a b n 2$ (yxiA), encoding a Bacillus subtilis GH43 arabinanase, Abn2, and its role in arabino-polysaccharide degradation. J. Bacteriol. 190: 4272-4280.

19. Park JM, Han NS, Kim TJ. 2007. Rapid detection and isolation of known and putative $\alpha$-L-arabinofuranosidase genes using degenerate PCR primers. J. Microbiol. Biotechnol. 17: 481-489.

20. Park JM, Jang MU, Kang JH, Kim MJ, Lee SW, Song YB, et al. 2012. Detailed modes of action and biochemical characterization of endo-arabinanase from Bacillus licheniformis DSM13. J. Microbiol. 50: 1041-1046.

21. Miller GL. 1959. Use of dinitrosalicyclic acid reagent for determination of reducing sugars. Anal. Chem. 31: 426-428.

22. Fan B, Blom J, Klenk HP, Borriss R. 2017. Bacillus amyloliquefaciens, Bacillus velezensis, and Bacillus siamensis form an "Operational group B. amyloliquefaciens" within the B. subtilis species complex. Front. Microbiol. 8: 22.

23. Fan B, Wang C, Song X, Ding X, Wu L, Wu H, et al. 2018. Bacillus velezensis FZB42 in 2018: The Gram-positive model strain for plant growth promotion and biocontrol. Front. Microbiol. 9: 2491.

24. Chen L, Gu W, Xu HY, Yang GL, Shan XF, Chen G. 2018. Comparative genome analysis of Bacillus velezensis reveals a potential for degrading lignocellulosic biomass. 3 Biotech. 8: 253.

25. Chen XH, Koumoutsi A, Scholz R, Eisenreich A, Schneider K, Heinemeyer I, et al. 2007. Comparative analysis of the complete genome sequence of the plant growth-promoting bacterium Bacillus amyloliquefaciens FZB42. Nat. Biotechnol. 25: 1007-1014.

26. Miyazaki K. 2005. Hyperthermophilic $\alpha$-L-arabinofuranosidase from Thermotoga maritima MSB8: molecular cloning, gene expression, and characterization of the recombinant protein. Extremophiles 9: 399-406.

27. Dumbrepatil A, Park JM, Jung TY, Song HN, Jang MU, Han NS, et al. 2012. Structural analysis of $\alpha$-L-arabinofuranosidase from Thermotoga maritima reveals characteristics for thermostability and substrate specificity. J. Microbiol. Biotechnol. 22: 17241730.

28. Lim YR, Yoon RY, Seo ES, Kim YS, Park CS, Oh DK. 2010. Hydrolytic properties of a thermostable $\alpha$-L-arabinofuranosidase from Caldicellulosiruptor saccharolyticus. J. Appl. Microbiol. 109: 1188-1197. 